\title{
Efficient Control of Atom Arrangement in Ternary Metal Chal- cogenide Nanoparticles Using Precursor Oxidation State
}

Emily A. Eikey ${ }^{\dagger}$, Xing Yee Gan ${ }^{\dagger}$, Derrick C. Kaseman ${ }^{\dagger}$, Corban G. E. Murphey ${ }^{\dagger}$, Scott E. Crawford $^{\dagger}$, Kathryn A. Johnston ${ }^{\dagger}$, Sadegh Yazdi ${ }^{\ddagger}$, and Jill E. Millstone ${ }^{*,+, \S, \|}$

tDepartment of Chemistry, University of Pittsburgh, 219 Parkman Avenue, Pittsburgh, Pennsylvania 15260, United States

*Renewable and Sustainable Energy Institute, University of Colorado at Boulder, 4001 Discovery Drive, Boulder, Colorado 80309, United States

§Department of Chemical and Petroleum Engineering, University of Pittsburgh, 3700 O'Hara Street, Pittsburgh, Pennsylvania 15261, United States

\|Department of Mechanical Engineering and Materials Science, University of Pittsburgh, 3700 O'Hara Street, Pittsburgh, Pennsylvania 15261, United States

\section{Contents}

Cu Concentration Estimate in $\mathrm{Cu}_{2-x}$ Se NPs for CE

Figure S1. Calibration curve and equation used to estimate the moles of $\mathrm{Cu}$ in a $\mathrm{Cu}_{2-x} \mathrm{Se} \mathrm{NP}$ sample for CE using the LSPR maximum intensity

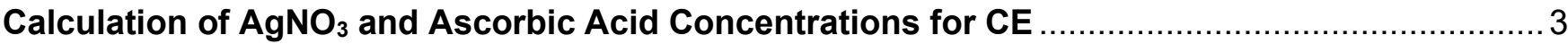

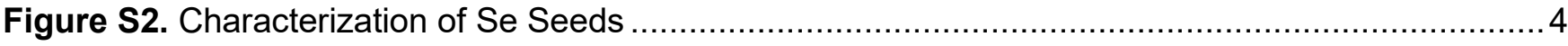

Figure S3. Size distributions for Cu/Ag/Se NPs synthesized via the one-pot and CE techniques ..........4

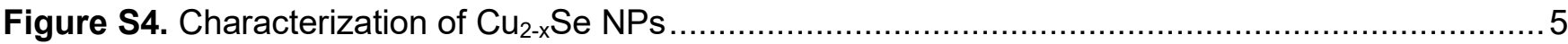

Table S1. Similar Percentages of Selenium in the Final NP Samples Regardless of Cu and Ag Contents or Synthetic Method as Determined by ICP-OES

Figure S5. Characterization of coalesced $\mathrm{Ag}_{2} \mathrm{Se} \mathrm{NPs}$.

Figure S6. Linescans generated from STEM-EDS maps for Cu/Ag/Se NPs synthesized via the one-pot and $\mathrm{CE}$ techniques

Figure S7. Representative Se Ka STEM-EDS maps for Cu/Ag/Se NPs synthesized via the one-pot and CE techniques

Figure S8. Collection of representative STEM-EDS maps for Cu/Ag/Se NPs synthesized via the original one-pot, CE, and modified one-pot techniques.

Role of Reducing Agent on Se Seeds: Discussion

Figure S9. XPS of the Se 3d region for untreated Se seeds, Se seeds treated with ascorbic acid, Se seeds treated with $\mathrm{NaBH}_{4}$, and $\mathrm{Cu}_{2-x} \mathrm{Se} \mathrm{NPs}$

Mixture of $\mathrm{Cu}$ Oxidation States Yields a Distribution of $\mathrm{Cu}$ :Se Stoichiometries in the Original One-Pot Synthesis

Figure S10. Characterization of $\mathrm{Cu}_{1.8} \mathrm{Se}$ NPs 
Figure S11. PXRD patterns for $\mathrm{Cu}_{1.8} \mathrm{Se} N P s, \mathrm{Cu}_{1.3} \mathrm{Se} N P s$, and $20 \% \mathrm{Ag}$ content $\mathrm{Cu} / \mathrm{Ag} / \mathrm{Se}$ NP sample

synthesized via the original one-pot technique 13

Synthesis of $\mathrm{Cu}_{1.8} \mathrm{Se}$ NPs 13

Figure S12. Size distribution for $\mathrm{Cu} / \mathrm{Ag} / \mathrm{Se}$ NPs synthesized via the modified one-pot technique 14

Figure S13. Linescans generated from STEM-EDS maps for Cu/Ag/Se NPs synthesized via the modified one-pot technique

Figure S14. Representative Se Ka STEM-EDS maps for Cu/Ag/Se NPs synthesized via the original one-pot, CE, and modified one-pot techniques at similar Ag contents 15

References 


\section{Cu Concentration Estimate in $\mathrm{Cu}_{2-x} \mathrm{Se}$ NPs for CE}

To semi-quantitatively assess the $\mathrm{Cu}$ concentrations in $\mathrm{Cu}_{2-x} \mathrm{Se}$ NP stock solutions to be used for CE experiments, a combination of extinction spectroscopy and ICP-OES was used to construct a calibration curve (Figure S1). Extinction spectra and ICP-OES measurements were collected as described in the Experimental Section. To construct the calibration curve, four $\mathrm{Cu}_{2-x} \mathrm{Se}$ NP batches were synthesized and purified, resulting in $\mathrm{Cu}_{2-x} \mathrm{Se}$ NP stock solutions. Extinction measurements of diluted aliquots of the $\mathrm{Cu}_{2-x} \mathrm{Se} \mathrm{NP}$ stock solutions were collected to determine the localized surface plasmon resonance (LSPR) maximum intensities near $1050 \mathrm{~nm}$. Each of those diluted aliquots were then separately digested with $5 \%$ aqua regia, and the Cu concentrations were determined by ICP-OES. The calibration curve was fit to a linear trendline and an equation was derived, which was used to estimate the Cu concentration in each $\mathrm{Cu}_{2-x} \mathrm{Se} \mathrm{NP}$ sample to be used for subsequent CE syntheses.

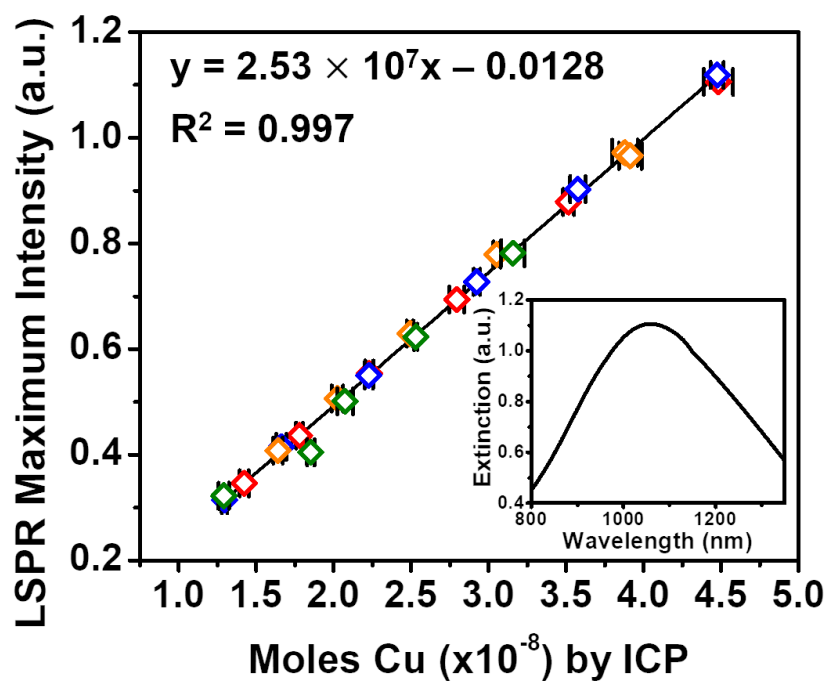

Figure S1. Calibration curve and equation used to estimate the moles of $\mathrm{Cu}$ in a $\mathrm{Cu}_{2-\mathrm{x}} \mathrm{Se} \mathrm{NP}$ sample for CE using the LSPR maximum intensity near $1050 \mathrm{~nm}$. Four separate syntheses are represented by color, each with at least 5 different $\mathrm{Cu}_{2-x} \mathrm{Se}$ NP concentrations. The error bars represent the standard deviation of a single sample measured 3 times by ICP-OES. A representative extinction spectrum of a $\mathrm{Cu}_{2-x} \mathrm{Se} \mathrm{NP}$ sample depicting the LSPR feature is shown in the inset.

\section{Calculation of $\mathrm{AgNO}_{3}$ and Ascorbic Acid Concentrations for CE}

The $\mathrm{Cu}$ concentration of a $\mathrm{Cu}_{2-x} \mathrm{Se}$ NP sample to be used for $\mathrm{CE}$ was estimated using the calibration curve and equation shown above, and the molar excess of $\mathrm{Ag}$ to be added was determined based on the extent of CE desired, which was varied in this work from 2.6 to $10.3 \mathrm{Ag}: \mathrm{Cu}$. Once the moles of $\mathrm{Ag}$ to be added was calculated, the moles of ascorbic acid needed was then determined using a molar ratio of 1.4:1 ascorbic acid: $\mathrm{AgNO}_{3}$. This ratio remained constant regardless of the extent of $\mathrm{CE}$ and was based on the synthesis of $\mathrm{Cu}_{2-x} \mathrm{Se} \mathrm{NPs}$, which uses a molar ratio of $1.4: 1$ ascorbic acid:CuSO $\mathrm{C}_{4} \cdot 5 \mathrm{H}_{2} \mathrm{O}$. 


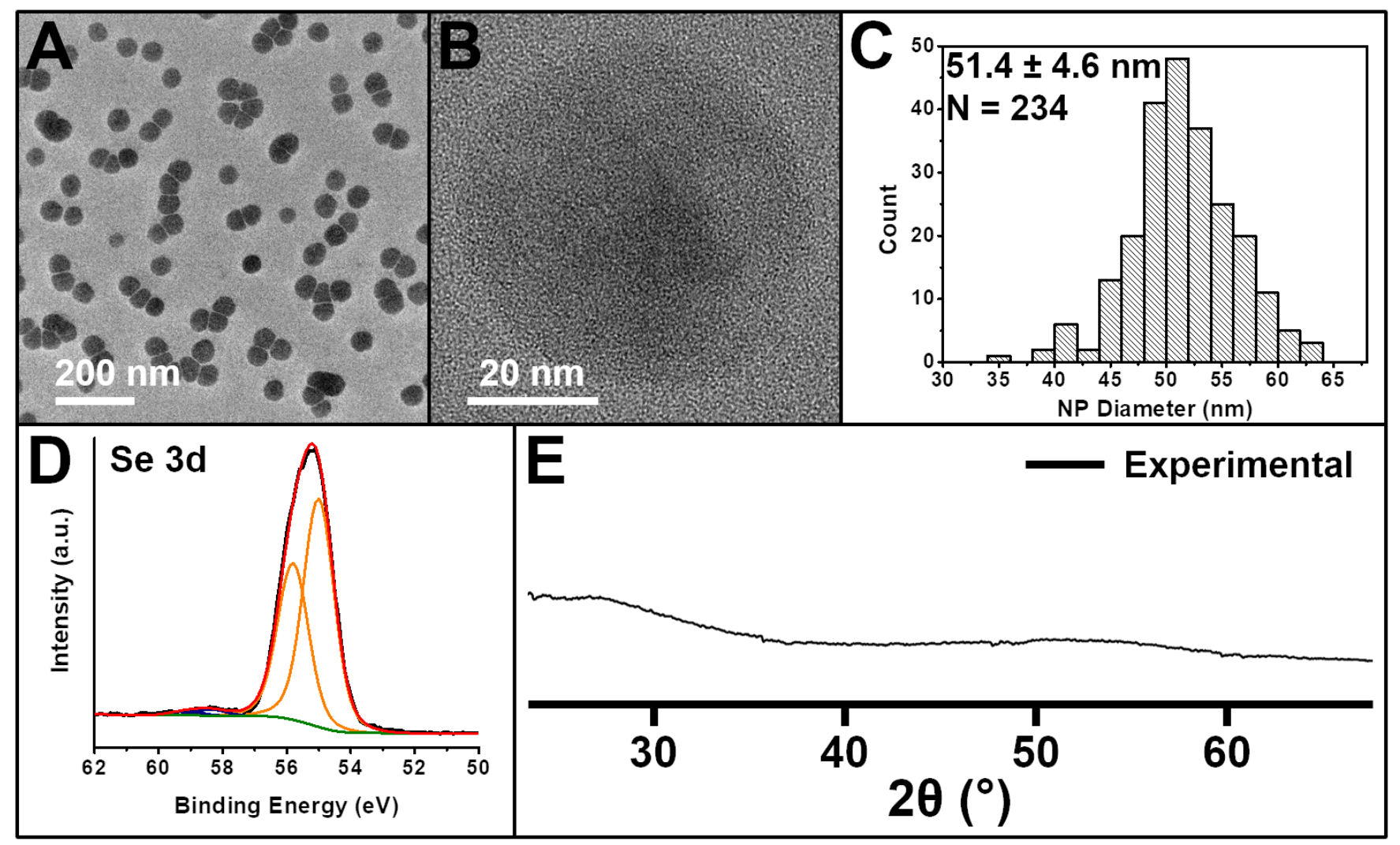

Figure S2. Representative (A and B) TEM images and (C) size distribution of Se seeds. (D) XPS of the Se $3 d$ region and $(E)$ experimental PXRD pattern of Se seeds, indicating that the $\mathrm{Se}^{0} \mathrm{NPs}$ are primarily amorphous. XPS of the Se 3d region shows the experimental spectrum in black, the $\mathrm{Se}^{0}$ fit in orange, the $\mathrm{Se}^{4+}$ fit in dark blue, the overall fit in red, and the baseline in green.
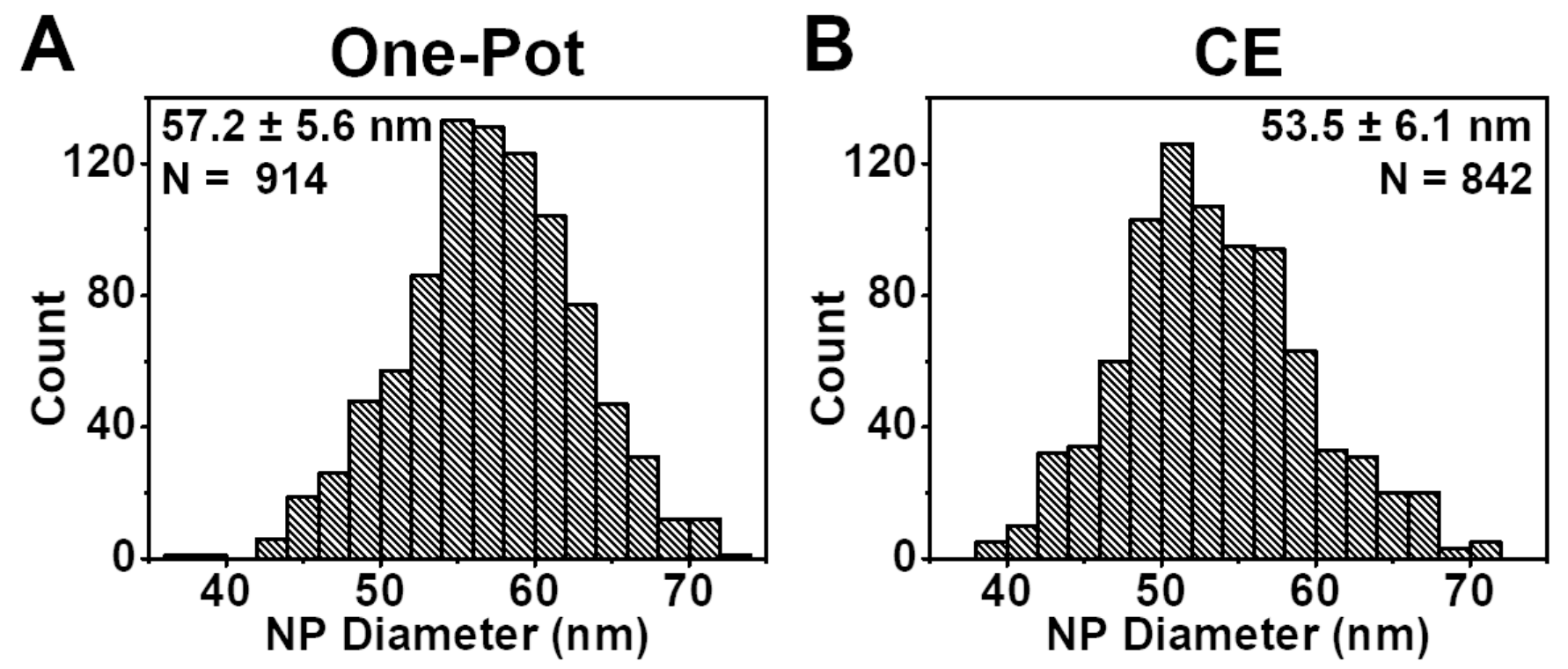

Figure S3. Size distributions for $\mathrm{Cu} / \mathrm{Ag} / \mathrm{Se} N \mathrm{~N}$ s synthesized via the (A) one-pot and (B) CE techniques indicating that the NP diameters do not change as a function of synthetic method. Distributions represent NP diameters over the range of compositions investigated, not for a single Ag content, suggesting that the resulting NP diameters do not change as a function of Ag content. 


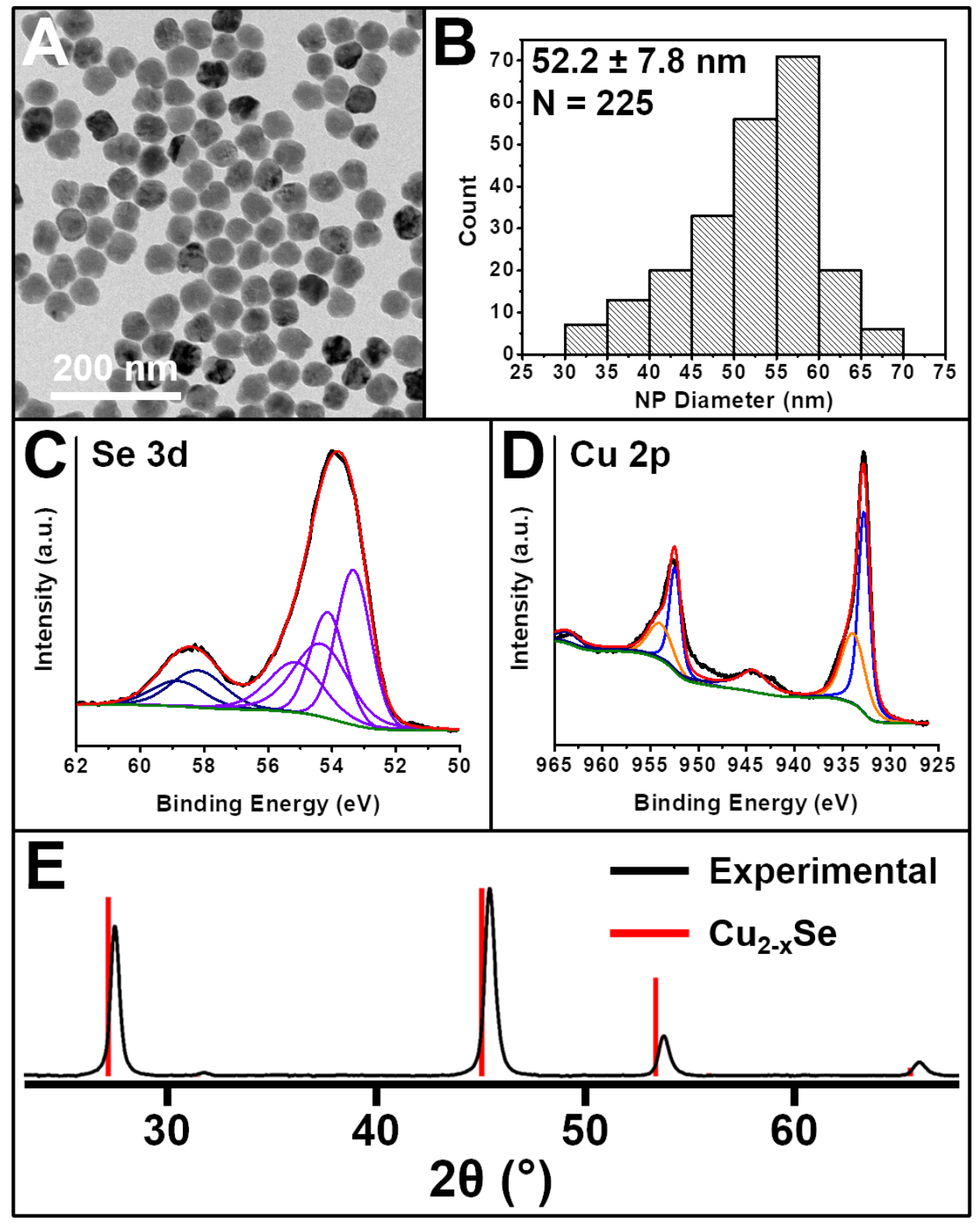

Figure S4. Representative (A) TEM image and (B) size distribution of $\mathrm{Cu}_{2-x} \mathrm{Se}$ NPs. (C) XPS of the Se $3 d$ and $(D)$ Cu $2 p$ regions. XPS of the Se $3 d$ region shows the experimental spectrum in black, the $\mathrm{Se}^{2-}$ fits in purple, the $\mathrm{Se}^{4+}$ fit in dark blue, the overall fit in red, and the baseline in green. XPS of the Cu $2 p$ region shows the experimental spectrum in black, the $\mathrm{Cu}^{1+}$ fit in blue, the $\mathrm{Cu}^{2+}$ fit in orange, the satellite fit in dark blue, the overall fit in red, and the baseline in green and indicates that there is a mixture of $\mathrm{Cu}^{1+}$ and $\mathrm{Cu}^{2+}$ cations. XPS of the Se 3d region indicates that the majority of Se atoms are in the 2- oxidation state in slightly different environments due to a mixture of $\mathrm{Cu}^{1+}$ and $\mathrm{Cu}^{2+}$. It should be noted that the fits indicate the environments present, but are not quantitative. (E) Experimental PXRD pattern of $\mathrm{Cu}_{2-x} \mathrm{Se}$ NPs (black) closely matches the $\mathrm{Cu}_{2-x}$ Se database pattern (red, PDF no. 00-046-1129), indicating that the NPs have a cubic crystallographic phase. 
Table S1. Similar Percentages of Selenium in the Final NP Samples Regardless of Cu and Ag Contents or Synthetic Method as Determined by ICP-OES

\begin{tabular}{|c|c|c|c|c|c|}
\hline \multirow{2}{*}{ Method } & $\begin{array}{c}\text { Percentages from Total of } \\
\text { Cu and Ag Only }\end{array}$ & \multicolumn{3}{|c|}{$\begin{array}{c}\text { Percentages from Total of } \\
\text { Cu, Ag, and Se }\end{array}$} \\
\cline { 2 - 6 } & $\% \mathrm{Cu}$ & $\% \mathrm{Ag}$ & $\% \mathrm{Cu}$ & $\% \mathrm{Ag}$ & $\% \mathrm{Se}$ \\
\hline \multirow{2}{*}{ One-Pot } & $87.5 \pm 1.5$ & $12.5 \pm 1.5$ & $55.7 \pm 1.0$ & $8.00 \pm 1.0$ & $36.3 \pm 0.81$ \\
\cline { 2 - 6 } & $20.5 \pm 1.0$ & $79.5 \pm 1.0$ & $13.7 \pm 0.52$ & $53.3 \pm 1.5$ & $33.0 \pm 1.5$ \\
\hline \multirow{2}{*}{$\mathrm{CE}$} & $76.3 \pm 1.5$ & $23.7 \pm 1.5$ & $48.4 \pm 0.76$ & $15.1 \pm 1.0$ & $36.5 \pm 0.32$ \\
\cline { 2 - 6 } & $25.8 \pm 0.51$ & $74.2 \pm 0.51$ & $16.8 \pm 0.28$ & $48.2 \pm 0.56$ & $35.0 \pm 0.56$ \\
\hline
\end{tabular}

${ }^{*}$ All standard deviations were determined from at least 5 independent syntheses.

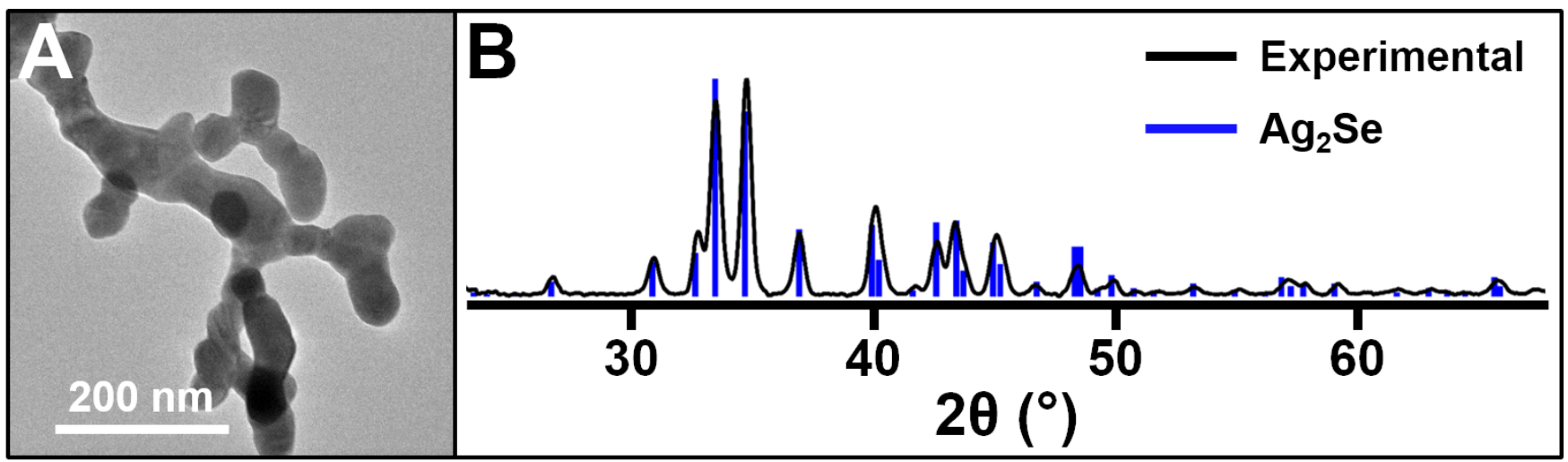

Figure S5. Representative (A) TEM image and (B) experimental PXRD pattern of coalesced $\mathrm{Ag}_{2} \mathrm{Se}$ NPs synthesized via the one-pot method (black), which closely matches the $\mathrm{Ag}_{2} \mathrm{Se}$ database pattern (blue, PDF no. 00-024-1041), indicating that the NPs have an orthorhombic crystallographic phase. 

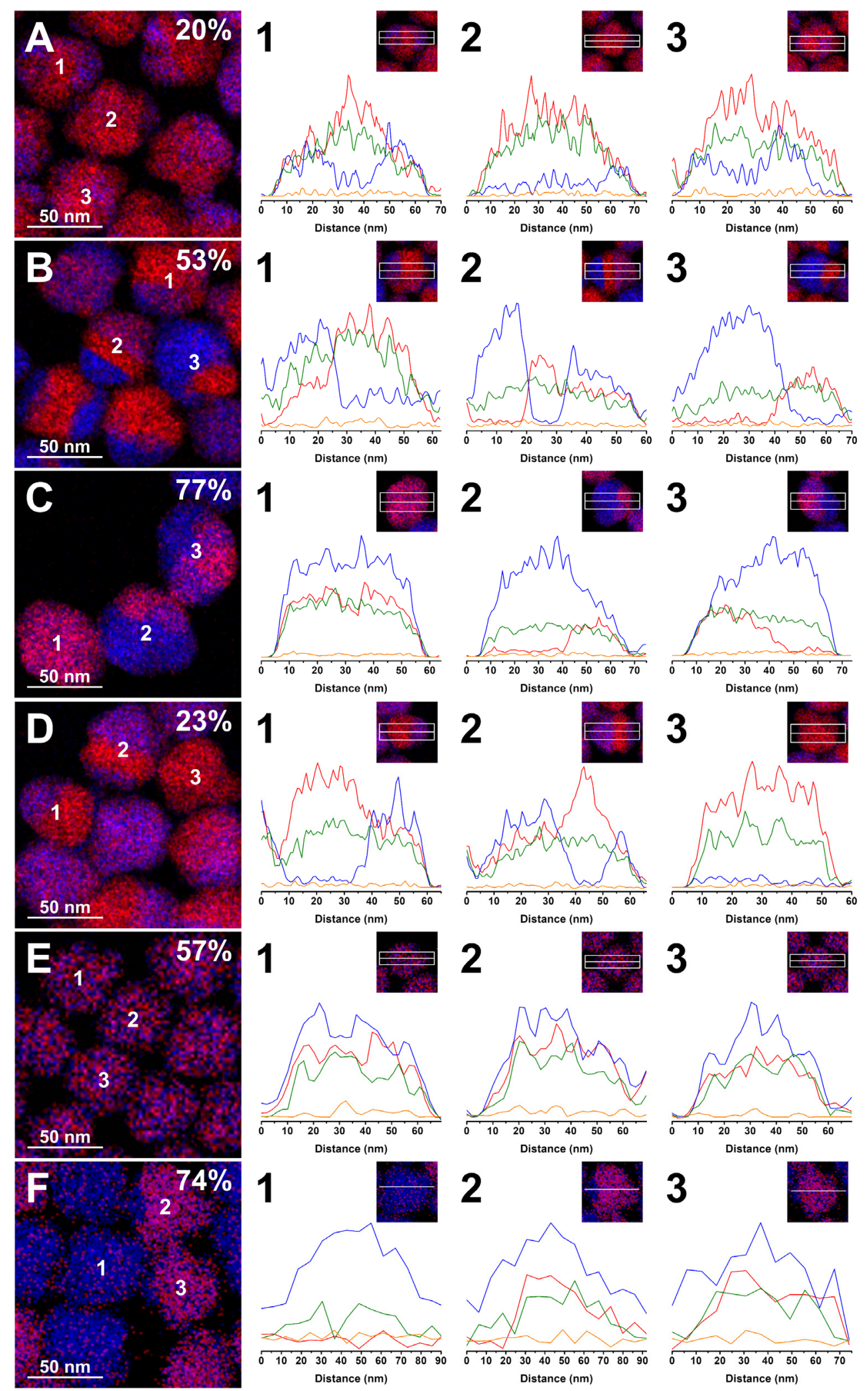

Figure S6. Linescans generated from STEM-EDS maps in Figures 3 and $\mathbf{S 7}$ for Cu/Ag/Se NPs synthesized via the one-pot technique at (A) 20\%, (B) 53\%, and (C) $77 \%$ Ag contents and CE technique at (D) 23\%, (E) 57\%, and (F) 74\% Ag contents, each determined by ICP. Cu Ka, Ag La, Se Ka, and Eu La signals are represented in red, blue, green, and orange, respectively. Eu is used as a baseline. Linescan widths represent the pixel range used to generate the linescans. (F) Linescans for the sample with $74 \%$ $\mathrm{Ag}$ content were collected on a different electron microscope and were generated with a bin factor of 8 . 

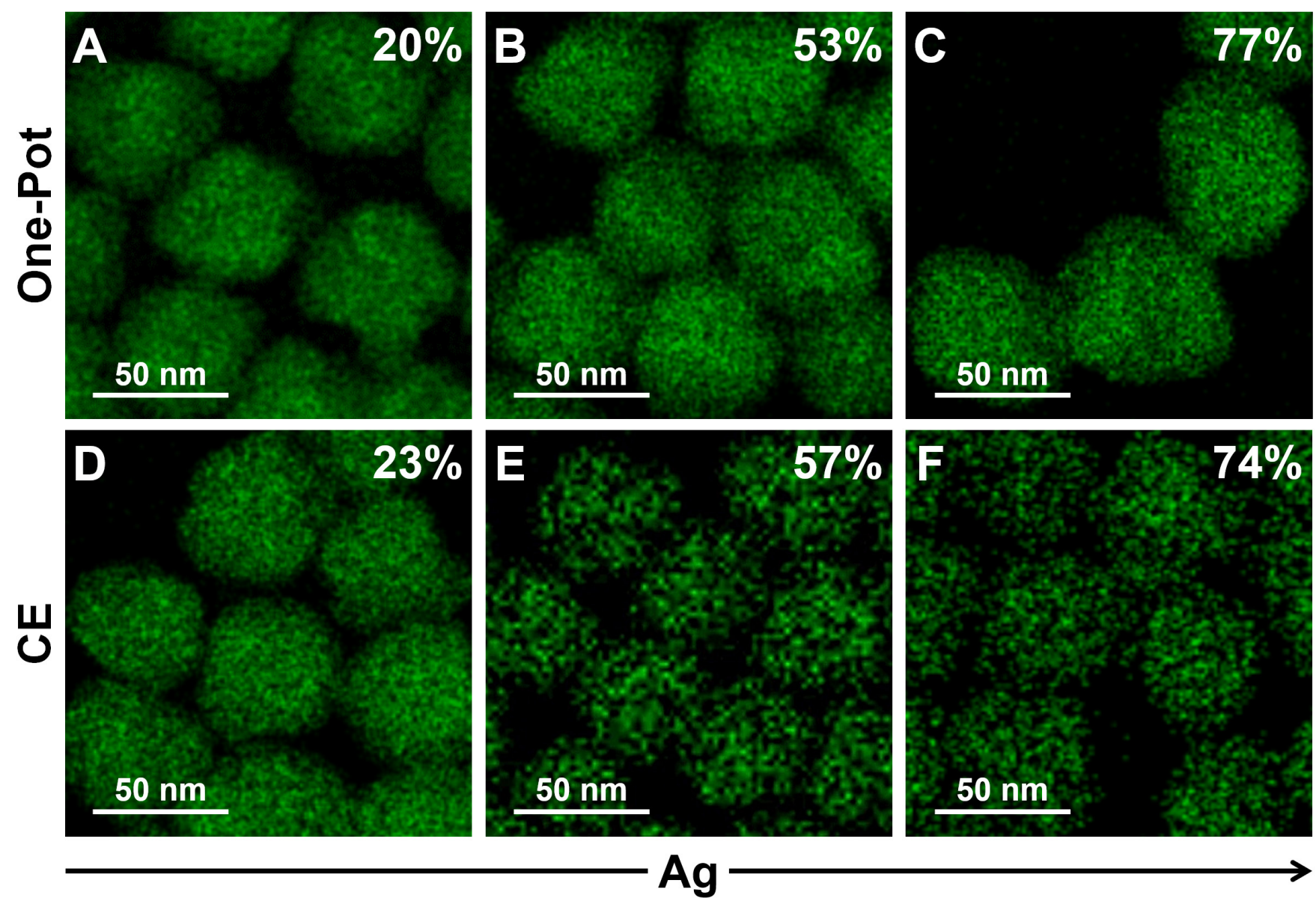

Figure S7. Representative STEM-EDS maps for Cu/Ag/Se NPs synthesized via the one-pot technique at (A) $20 \%$, (B) $53 \%$, and (C) $77 \%$ Ag contents and CE technique at (D) $23 \%$, (E) $57 \%$, and (F) $74 \% \mathrm{Ag}$ contents, each determined by ICP. Linescans of individual particles can be found in Figures 6 and S6. Se Ka signal is represented in green. (The STEM-EDS map for (F) sample with $74 \% \mathrm{Ag}$ content accessed via CE was collected on a different electron microscope using different software (see Experimental Section for details); thus any differences in contrast should not be taken as quantitative.) 


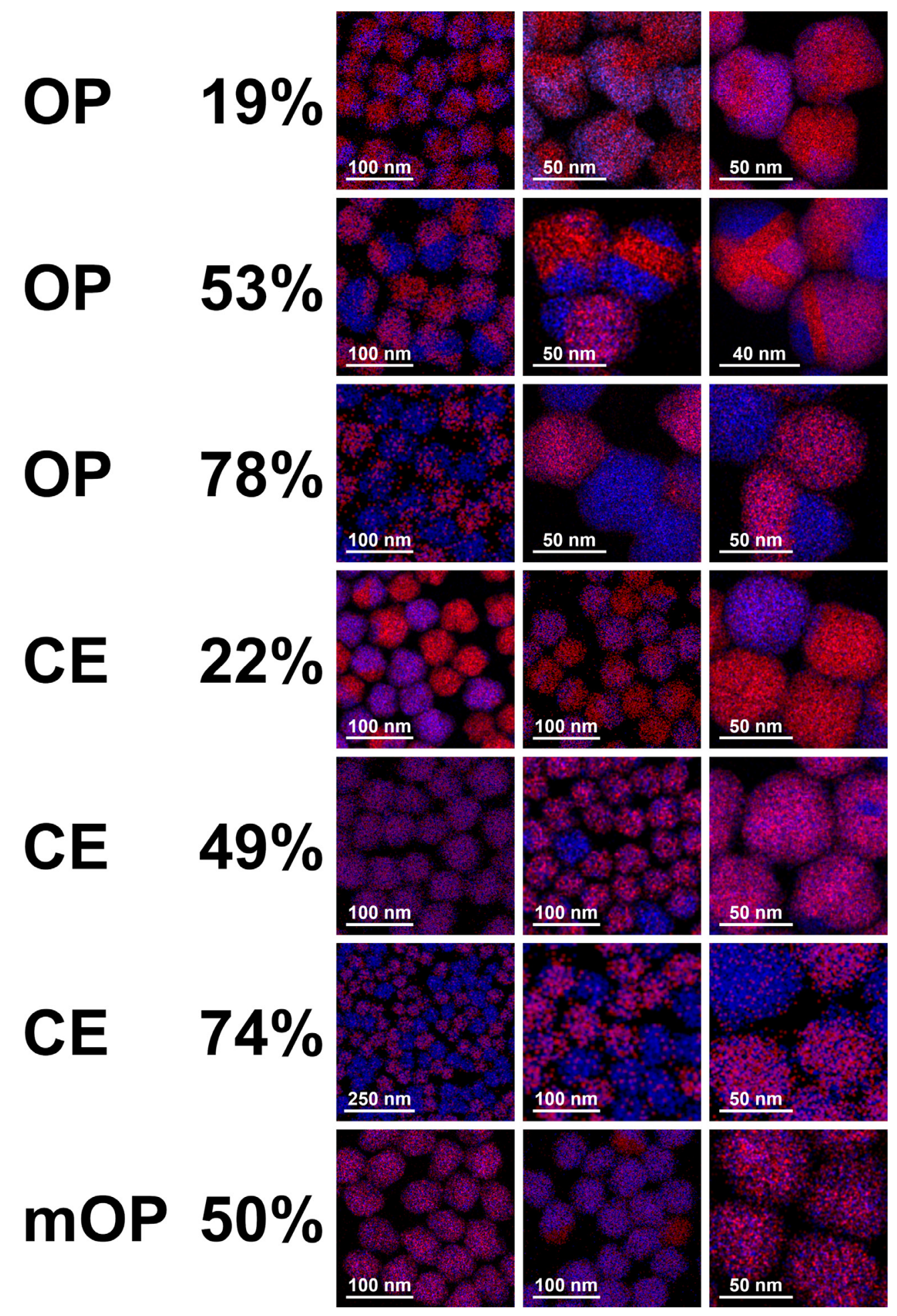

Figure S8. Collection of representative STEM-EDS maps for Cu/Ag/Se NPs synthesized via the one-pot (OP), $\mathrm{CE}$, and modified one-pot (mOP) techniques with average Ag contents listed, some of which were used to generate the histogram of morphology distributions in Figure 4. Reported Ag contents are the average of two independent synthetic trials, and the standard deviations for the averaged $\mathrm{Ag}$ contents are as follows: $19 \pm 2 \%, 53 \pm 0 \%, 78 \pm 1 \%, 22 \pm 2 \%, 49 \pm 11 \%, 74 \pm 0 \%$, and $50 \pm 2 \%$. Cu Ka signal is represented in red, Ag La signal is represented in blue, and Se Ka signal is not shown here for clarity. (The STEM-EDS maps were collected on two different electron microscopes using different software (see Experimental Section for details); thus any differences in contrast should not be taken as quantitative.) 


\section{Role of Reducing Agent on Se Seeds: Discussion}

During the one-pot synthesis, the Se seeds are synthesized, followed by the addition of the cation(s) of interest and ascorbic acid. We investigated the interaction of the reducing agent (ascorbic acid) with these Se seeds using XPS. First, we compared the Se 3d XPS region of untreated Se seeds, Se seeds treated with ascorbic acid, Se seeds treated with $\mathrm{NaBH}_{4}$, and $\mathrm{Cu}_{2-\mathrm{S}} \mathrm{Se} \mathrm{NPs}$ (Figure S9). For both experiments using reducing agent, the same amount of reducing agent that is added following Cu and $\mathrm{Ag}$ precursor addition in the one-pot synthesis was added. The Se 3d region for the untreated Se seeds shows a peak near $55.2 \mathrm{eV}$, suggesting that the majority of $\mathrm{Se}$ atoms are in the $\mathrm{Se}^{0}$ oxidation state. ${ }^{1}$ For the Se seeds treated with ascorbic acid, a shift in the center of mass of the peak to $54.8 \mathrm{eV}$ is observed, suggesting that while the majority of $\mathrm{Se}$ atoms remain in the $\mathrm{Se}^{0}$ oxidation state, a population of $\mathrm{Se}$ atoms have been reduced to $\mathrm{Se}^{2-}$. For the Se seeds treated with $\mathrm{NaBH}_{4}$, the Se 3d peak shifted to 54.6 $\mathrm{eV}$, suggesting that while similar to the ascorbic acid case, more Se atoms have been reduced to $\mathrm{Se}^{2-}$. This observation is expected, as $\mathrm{NaBH}_{4}$ is a stronger reducing agent than ascorbic acid. ${ }^{2}$ Still, the shifts to lower binding energy with the addition of reducing agent were not as significant as the shift observed for the $\mathrm{Cu}_{2-x} \mathrm{Se} N P s$. For the $\mathrm{Cu}_{2-x} \mathrm{Se} N P s$, the Se $3 \mathrm{~d}$ peak is observed near $53.9 \mathrm{eV}$, which is indicative of Se atoms in the 2- oxidation state. Additionally, another peak is observed at higher binding energy $(\sim 58.5 \mathrm{eV})$, suggesting that a portion of the Se atoms have oxidized, likely due to some of the Se atoms residing near the surface of the NPs. The appearance of these two peaks in the Se $3 \mathrm{~d}$ region for $\mathrm{Cu}_{2-\mathrm{x}} \mathrm{Se}$ NPs has been previously reported. ${ }^{3}$

The fitted XPS data gives an indication of the Se environments present, but it should be noted that these fits are not quantitative. The fits show that a single Se environment is present for the untreated Se seeds, two are present for the Se seeds treated with reducing agents (due to $\mathrm{Se}^{0}$ and $\mathrm{Se}^{2-}$ environments), and three are present for the $\mathrm{Cu}_{2-x} \mathrm{Se} \mathrm{NPs}$ (two due to $\mathrm{Se}^{2-}$ environments near $\mathrm{Cu}^{1+}$ and $\mathrm{Cu}^{2+}$, consistent with the XPS of the $\mathrm{Cu} 2 \mathrm{p}$ region (Figure S4D) and one due to a Se ${ }^{4+}$ environment). Thus, the clear shift of the center of mass of the Se 3d peak to lower binding energy indicates that when reducing agent is added, some fraction of $\mathrm{Se}^{0}$ is reduced to $\mathrm{Se}^{2-}$, yet this shift is not as significant as that observed for the $\mathrm{Cu}_{2-\mathrm{x}} \mathrm{Se} \mathrm{NPs}$, where Se atoms are in the 2- oxidation state. In all, the XPS experiments suggest that the reducing agent's main role is to reduce the metal precursor rather than the Se atoms, which are likely reduced by monovalent cations in solution. 


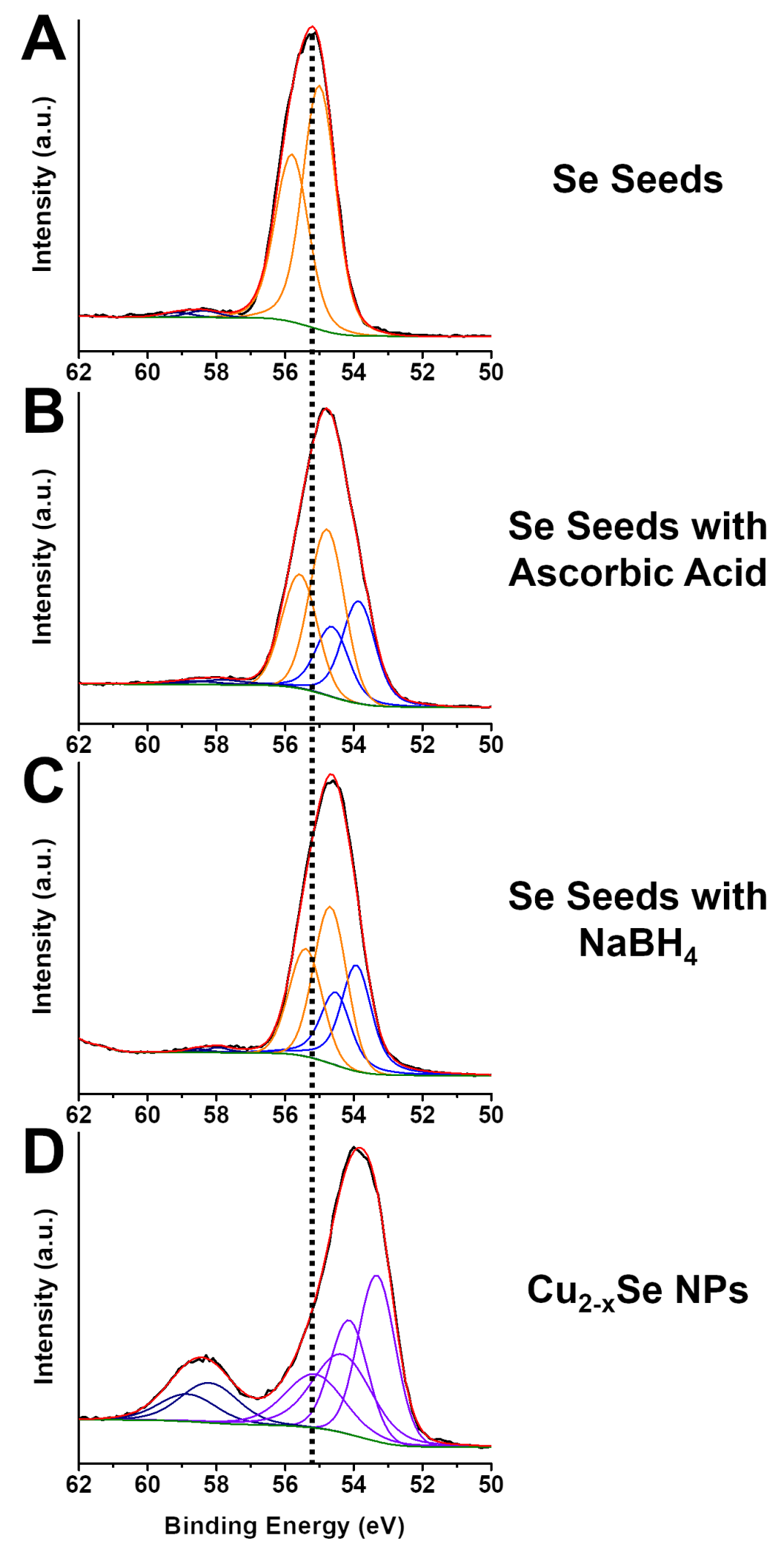

Figure S9. XPS of the Se 3d region for (A) untreated Se seeds, (B) Se seeds treated with ascorbic acid, (C) Se seeds treated with $\mathrm{NaBH}_{4}$, and (D) $\mathrm{Cu}_{2-x} \mathrm{Se}$ NPs. XPS shows the experimental spectrum in black, the $\mathrm{Se}^{0}$ fit in orange, the $\mathrm{Se}^{2-}$ fits in blue or purple, the $\mathrm{Se}^{4+}$ fit in dark blue, the overall fit in red, and the baseline in green. It should be noted that the fits indicate the environments present, but the fits are not quantitative. The black dotted line acts as a guide for the eye. 


\section{Mixture of Cu Oxidation States Yields a Distribution of $\mathrm{Cu}$ :Se Stoichiometries in the Original One- Pot Synthesis}

As stated in the main text, it is likely that because a mixture of $\mathrm{Cu}^{1+/ 2+}$ cations are present in the original one-pot synthesis, the resulting NPs have a distribution of $\mathrm{Cu}$ :Se stoichiometries in addition to other chemically-distinct crystallographic phases (i.e., CuAgSe or $\mathrm{Ag}_{2} \mathrm{Se}$ ), as indicated by the breadth in the PXRD peaks at both $27^{\circ}$ and $45^{\circ}$ (Figure 2A).

To confirm that a mixture of $\mathrm{Cu}$ oxidation states can impact the final $\mathrm{Cu}$ :Se ratio, we separately synthesized two cubic $\mathrm{Cu}_{2-x} \mathrm{Se} \mathrm{NPs}$, one using a mixture of $\mathrm{Cu}^{1+/ 2+}$ cations and another using a solution enriched in $\mathrm{Cu}^{1+}$ by changing the amount of ascorbic acid added to the $\mathrm{CuSO}_{4} \cdot 5 \mathrm{H}_{2} \mathrm{O}$ precursor at a constant $\mathrm{CuSO}_{4} \cdot 5 \mathrm{H}_{2} \mathrm{O}$ concentration (synthesis protocol below). TEM analysis indicates that the resulting NPs are similar in diameter to each other (Figure S4A, S4B, and S10). Using ICP-OES, the Cu:Se ratios were determined to be 1.3:1, made using the mixture of $\mathrm{Cu}^{1+/ 2+}$ cations, and 1.8:1, made using an enriched $\mathrm{Cu}^{1+}$ solution. These ICP results indicate that the $\mathrm{Cu}$ oxidation states present in the precursor solution dictate the $\mathrm{Cu}$ :Se ratio in the final NP sample.

In the one-pot synthesis of $\mathrm{Cu} / \mathrm{Ag} / \mathrm{Se} \mathrm{NPs}$, a mixture of $\mathrm{Cu}^{1+/ 2+}$ cations are present along with $\mathrm{Ag}^{1+}$. Based on the above results, it is likely that a distribution of $\mathrm{Cu}$ :Se ratios can be found in the resulting $\mathrm{Cu} / \mathrm{Ag} / \mathrm{Se}$ NPs. A close look at the PXRD patterns for the one-pot synthesis reveals that broadening in the pattern near $\mathrm{Cu}_{2-x}$ Se reflections is observed, specifically near $27^{\circ}$ and $45^{\circ}$ (Figure 2A). To confirm that a distribution of $\mathrm{Cu}$ :Se ratios is the likely source of broadening, PXRD of the $\mathrm{Cu}_{1.3} \mathrm{Se}$ and $\mathrm{Cu}_{1.8} \mathrm{Se}$ NP samples were collected and indicate that both samples have a cubic crystal structure (Figure S11). The reflections are observed at slightly different angles, indicating that the lattice parameters for the two samples differ, likely due to differences in $\mathrm{Cu}$ deficiencies (i.e., $\mathrm{Cu}_{1.3} \mathrm{Se}$ supports more $\mathrm{Cu}$ deficiencies than $\mathrm{Cu}_{1.8} \mathrm{Se}$, leading to a smaller lattice parameter and thus appearing at higher angles than $\mathrm{Cu}_{1.8} \mathrm{Se}$, which is consistent with the PXRD data). $\mathrm{Cu}_{2-x} \mathrm{Se}$ NPs are known to support Cu-deficient cubic phases that can result in changes in peak position, as we observe. ${ }^{4-8}$ Both of these cubic $\mathrm{Cu}_{2-x} \mathrm{Se} N \mathrm{NP}$ samples could contribute to the broadened PXRD reflections.

In addition to the presence of cubic $\mathrm{Cu}_{2-\mathrm{x}}$ Se phases with different $\mathrm{Cu}$ :Se ratios, this synthesis also likely yields $\mathrm{Cu}$ :Se ratios smaller than 1.3:1. When approaching a 1:1 ratio, CuSe forms, which is no longer cubic in structure. ${ }^{9}$ Database patterns for hexagonal and monoclinic CuSe indicate that the presence of $\mathrm{CuSe}$ is also likely and may contribute to the broadened reflections observed and the increased intensity of the reflection observed near $31^{\circ}$ (Figure S11). These database patterns support the hypothesis that a distribution of phases forms with various $\mathrm{Cu}$ :Se ratios for the original one-pot synthesis in addition to other chemically-distinct crystallographic phases.

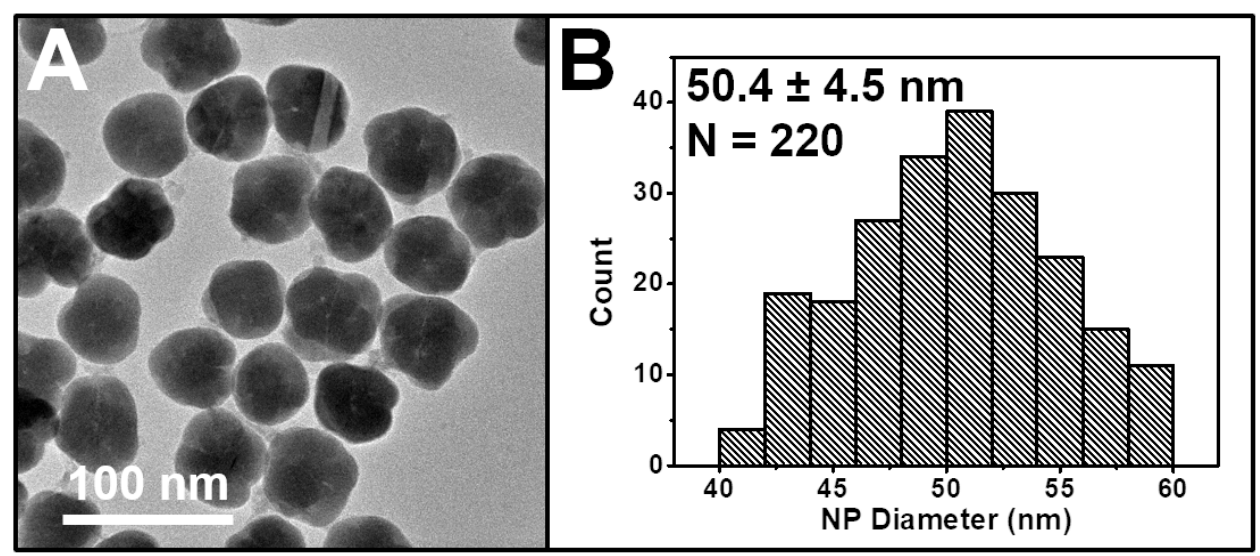

Figure S10. Representative (A) TEM image and (B) size distribution of $\mathrm{Cu}_{1.8} \mathrm{Se}$ NPs. 


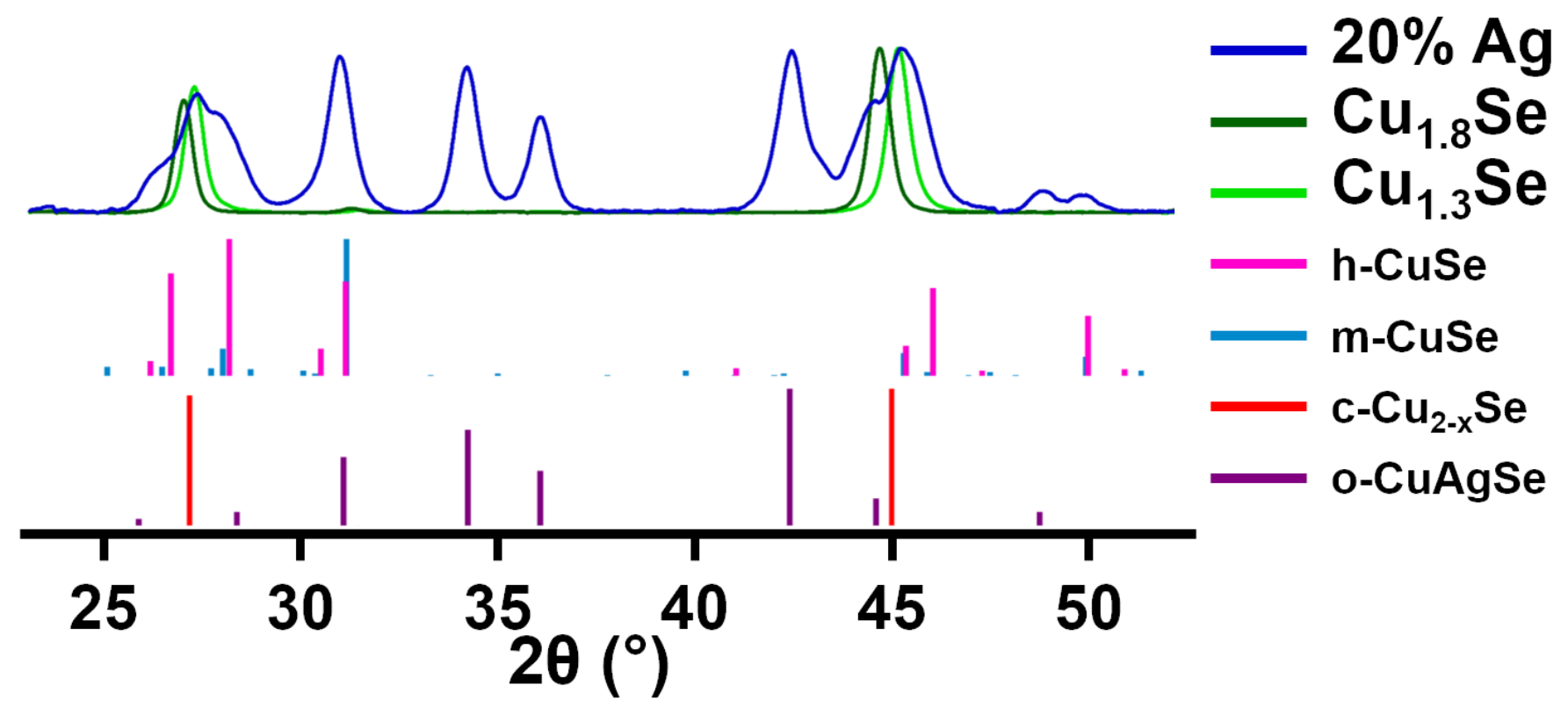

Figure S11. PXRD patterns for $\mathrm{Cu}_{1.8} \mathrm{Se} N P s, \mathrm{Cu}_{1.3} \mathrm{Se} N P s$, and $20 \%$ Ag content $\mathrm{Cu} / \mathrm{Ag} / \mathrm{Se} \mathrm{NP}$ sample synthesized via the original one-pot technique. Database patterns for hexagonal CuSe (h-CuSe, pink, PDF no. 00-034-0171), monoclinic CuSe (m-CuSe, light blue, PDF no. 00-049-1456), cubic Cu2-xSe (c$\mathrm{Cu}_{2-\mathrm{S}} \mathrm{Se}$, red, PDF no. 00-046-1129), and orthorhombic CuAgSe (o-CuAgSe, purple, PDF no. 00-0100451) are plotted. These control experiments support the formation of a distribution of $\mathrm{Cu}$ :Se ratios for the original one-pot method.

\section{Synthesis of $\mathrm{Cu}_{1.8} \mathrm{Se}$ NPs}

$\mathrm{Cu}_{1.8} \mathrm{Se} N \mathrm{~N} s$ were synthesized as the $\mathrm{Cu}_{2-x} \mathrm{Se} N P$ procedure indicates (see Experimental Section for details), save for a single modification. In this case, $500 \mu \mathrm{L}$ of $0.5 \mathrm{M} \mathrm{CuSO}_{4} \cdot 5 \mathrm{H}_{2} \mathrm{O}$ solution was combined with $3.09 \mathrm{~mL}$ of $1.15 \mathrm{M}$ ascorbic acid (an ascorbic acid:Cu molar ratio of 14.2:1) to give a yellow, enriched $\mathrm{Cu}^{1+}$ solution. It should be noted that the number of moles of $\mathrm{Cu}$ precursor does not change between two $\mathrm{Cu}_{2-x} \mathrm{Se}$ NP syntheses; however, the number of moles of ascorbic acid added does change significantly, which provides an enriched $\mathrm{Cu}^{1+}$ solution used to obtain $\mathrm{Cu}_{1.8} \mathrm{Se} \mathrm{NPs}$. This yellow, enriched $\mathrm{Cu}^{1+}$ solution was immediately added to the red Se seed solution. The NPs were left to stir for $\sim 16 \mathrm{~h}$ before purification, as described in the main text, to obtain purified $\mathrm{Cu}_{1.8} \mathrm{Se} \mathrm{NPs}$ for characterization. 


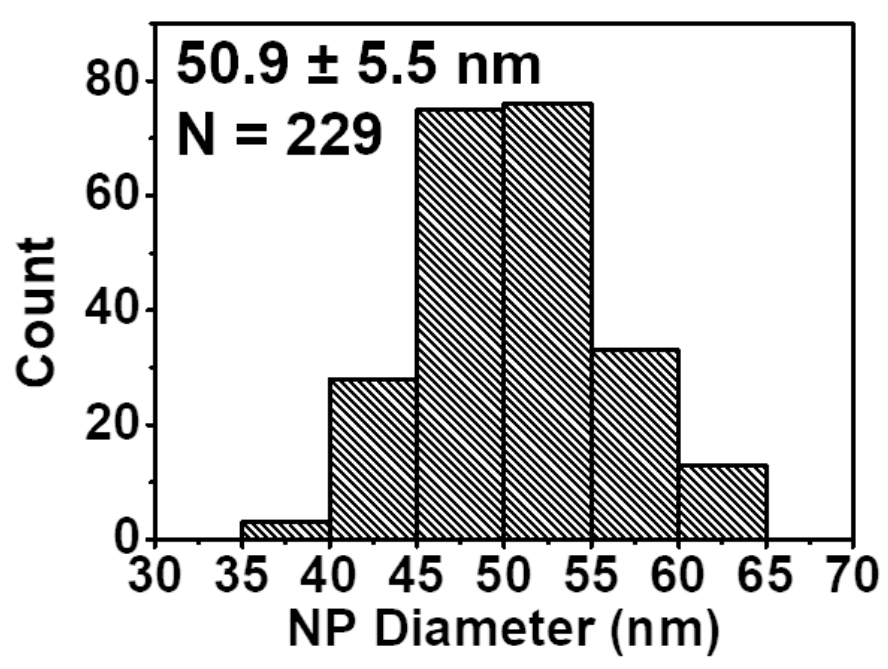

Figure S12. Size distribution for $\mathrm{Cu} / \mathrm{Ag} / \mathrm{Se}$ NPs synthesized via the modified one-pot technique.
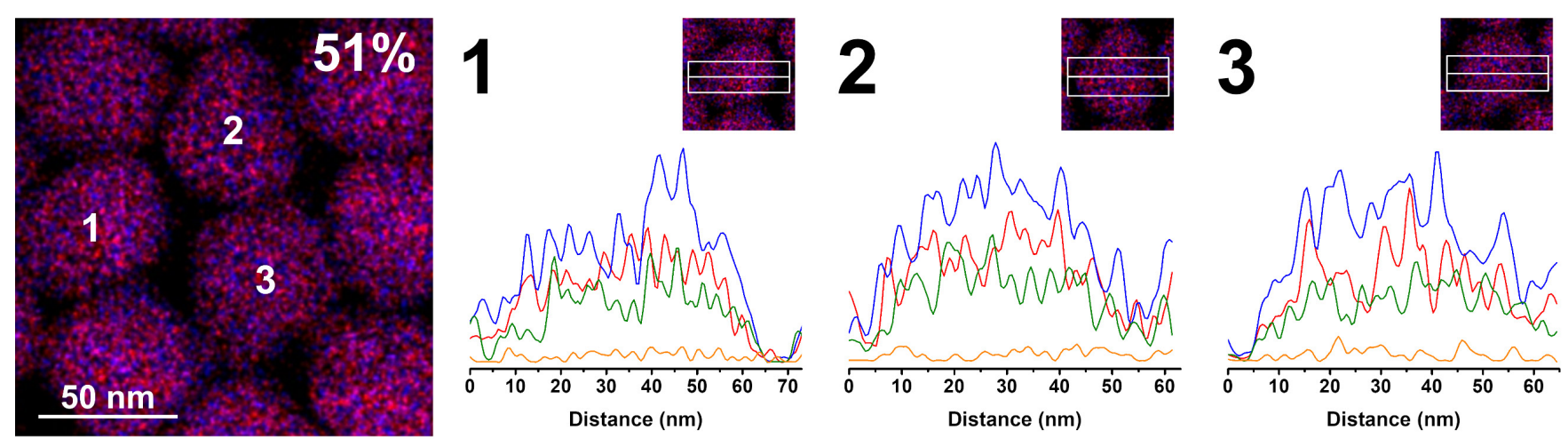

Figure S13. Linescans generated from the STEM-EDS maps in Figures 6 and S14 for Cu/Ag/Se NPs synthesized via the modified one-pot technique at 51\% Ag content as determined by ICP. Cu Ka, Ag La, Se Ka, and Eu La signals are represented in red, blue, green, and orange, respectively. Eu is used as a baseline. Linescan widths represent the pixel range used to generate the linescans. 

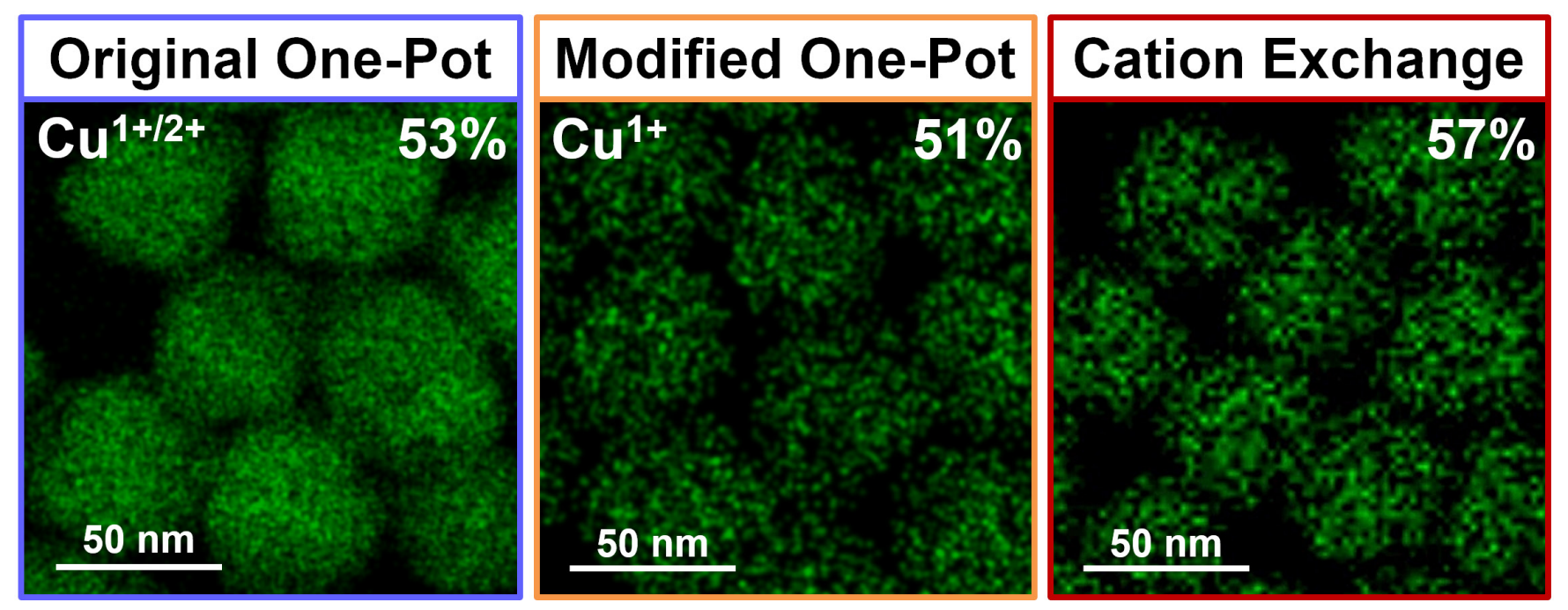

Figure S14. Representative STEM-EDS maps for Cu/Ag/Se NPs synthesized via the original one-pot, modified one-pot, and CE techniques at similar Ag contents as determined by ICP, where a mixture of $\mathrm{Cu}^{1+}$ and $\mathrm{Cu}^{2+}$ cations was used in the original one-pot technique and an enriched $\mathrm{Cu}^{1++}: \mathrm{Cu}^{2+}$ solution was used in the modified one-pot technique. Linescans of individual particles can be found in Figures $\mathbf{6}$, S6, and S13. Se Ka signal is represented in green.

\section{References}

1. Bahl, M. K.; Watson, R. L.; Irgolic, K. J. LMM Auger Spectra of Selenium and Some of its Compounds. J. Chem. Phys. 1980, 72, 4069-4077.

2. $\quad$ Rodrigues, T. S.; Zhao, M.; Yang, T.-H.; Gilroy, K. D.; da Silva, A. G. M.; Camargo, P. H. C.; Xia, Y. Synthesis of Colloidal Metal Nanocrystals: A Comprehensive Review on the Reductants. Chem. - Eur. J. 2018, 24, 16944-16963.

3. Riha, S. C.; Johnson, D. C.; Prieto, A. L. $\mathrm{Cu}_{2} \mathrm{Se}$ Nanoparticles with Tunable Electronic Properties Due to a Controlled Solid-State Phase Transition Driven by Copper Oxidation and Cationic Conduction. J. Am. Chem. Soc. 2011, 133, 1383-1390.

4. Marbella, L. E.; Gan, X. Y.; Kaseman, D. C.; Millstone, J. E. Correlating Carrier Density and Emergent Plasmonic Features in $\mathrm{Cu}_{2-x}$ Se Nanoparticles. Nano Lett. 2017, 17, 2414-2419.

5. Shafizade, R. B.; Ivanova, I. V.; Kazinets, M. M. Electron Diffraction Study of Phase Transformations of the Compound CuSe. Thin Solid Films 1978, 55, 211-220.

6. Kriegel, I.; Jiang, C.; Rodríguez-Fernández, J.; Schaller, R. D.; Talapin, D. V.; Da Como, E.; Feldmann, J. Tuning the Excitonic and Plasmonic Properties of Copper Chalcogenide Nanocrystals. J. Am. Chem. Soc. 2012, 134, 1583-1590.

7. Liu, X.; Swihart, M. T. Heavily-Doped Colloidal Semiconductor and Metal Oxide Nanocrystals: An Emerging New Class of Plasmonic Nanomaterials. Chem. Soc. Rev. 2014, 43, 3908-3920.

8. Agrawal, A.; Cho, S. H.; Zandi, O.; Ghosh, S.; Johns, R. W.; Milliron, D. J. Localized Surface Plasmon Resonance in Semiconductor Nanocrystals. Chem. Rev. 2018, 118, 3121-3207.

9. Heyding, R. D. The Copper/Selenium System. Can. J. Chem. 1966, 44, 1233-1236. 